\title{
An in-time damage identification approach based on the Kalman filter and energy equilibrium theory*
}

\author{
Xing-huai HUANG ${ }^{1}$, Shirley DYKE ${ }^{2}$, Zhao-dong XU ${ }^{\dagger 11}$ \\ $\left({ }^{1}\right.$ Key Laboratory of C\&PC Structures of the Ministry of Education, Southeast University, Nanjing 210096, China) \\ $\left({ }^{2}\right.$ School of Civil Engineering, Purdue University, West Lafayette IN 47907, USA) \\ †E-mail: xuzhdgyq@seu.edu.cn
}

Received June 5, 2014; Revision accepted Oct. 13, 2014; Crosschecked Jan. 12, 2015

\begin{abstract}
In research on damage identification, conventional methods usually face difficulties in converging globally and rapidly. Therefore, a fast in-time damage identification approach based on the Kalman filter and energy equilibrium theory is proposed to obtain the structural stiffness, find the locations of damage, and quantify its intensity. The proposed approach establishes a relationship between the structural stiffness and acceleration response by means of energy equilibrium theory. After importing the structural energy into the Kalman filter algorithm, unknown parameters of the structure can be obtained by comparing the predicted energy and the measured energy in each time step. Numerical verification on a highway sign support truss with and without damage indicates that the updated Young's modulus can converge to the true value rapidly, even under the effects of external noise excitation. In addition, the calculation time taken for each step of the approach is considerably shorter than the sampling period ( $1 / 256 \mathrm{~s})$, which means that, this approach can be implemented in-time and on-line.
\end{abstract}

Key words: In-time model updating, Kalman filter, Energy equilibrium theory, Damage identification, Anti-noise capacity, Structure health monitoring

doi: $10.1631 /$ jzus.A1400163

Document code: A

CLC number: TU192

\section{Introduction}

Highway sign support trusses are 3D assemblies of lightweight linear members, which make them efficient and cost-effective structures for long spans. This kind of structure is essential because it gives drivers instructions and information to avoid traffic accidents. Transportation departments have been designing and building overhead highway sign support trusses for a number of years in the United States

\footnotetext{
ॠCorresponding author

* Project supported by the National Science Foundation of USA (Nos. CNS-1035748 and CNS-1035773), the Program for Jiangsu Province 333 Talents, and the Funds for Excellent Doctoral Dissertation of Southeast University (No. YBJJ1207), China (DD ORCID: Xing-huai HUANG, http://orcid.org/0000-0001-9989-577X; Zhao-dong XU, http://orcid.org/0000-0003-0544-8253

(C) Zhejiang University and Springer-Verlag Berlin Heidelberg 2015
}

(Nadauld and Pantelides, 2007). These structures cover relatively long-spans and serve in harsh environments with erosion caused by water, wind, and ice, vibration caused by wind and vehicles passing by, and so on. These negative factors can induce damage to these structures, including fatigue on anchor bolts and connection plates, bending about the diagonal, and cracks initiating from welded joints (Park and Stallings, 2006). Such damage may cause failure of structural members and even collapse of the whole structure, with danger to people in passing vehicles. To quickly determine maintenance and repair schemes for engineers, it is essential to use a convenient, valid, and trusty method for quickly identifying damage on these structures. For this reason, usable damage identification methods are crucial and essential to this type of structure, which constantly experiences vibration. 
With the increasing need for non-destructive evaluation of structural reliability, a wide variety of damage identification methods have been developed in recent years. There are numerous different nondestructive methods for damage identification, which can be classed mainly in two groups: non-modelbased methods and model based methods. The nonmodel-based methods detect damage through vibrational signals directly without use of a structural model. The non-model-based methods mainly include methods based on the change in natural frequency (Lee and Chung, 2000; Kim et al., 2003), the change of structural vibration mode (Khoo et al., 2004), the change of structural flexibility or stiffness (Yan and Golinval, 2005), the transfer function (Park and Park, 2005), the statistic information (Iwasaki et al., 2004; López-Díez et al., 2005; Garcia-Perez et al., 2013), the power flow (Li et al., 2004), and so on. The model-based methods detect damage based on predetermined structural models, in which the model updating methods are widely used (Sinha and Friswell, 2003; Sadr et al., 2007; Zhao et al., 2009; Lei et al., 2012; Liu and Duan, 2012; Erdogan and Bakir, 2013; Liu et al., 2013). However, most of the above methods are not quick, which means they provide structural information with an obvious time delay caused by their massive and off-line calculation process. In contrast, in-time damage identification methods can improve the ability to detect damage by first decision-makers (e.g., authorities, engineers, fire fighting, policing, Federal Emergency Management Agency). Nevertheless, it has always been a challenging problem to identify damage in structures which contain a large number of degrees of freedom (DOFs). It is also a difficult issue to model and identify such large-size structural system and enable the identification procedure to execute in an on-line or real-time environment.

With the help of the Kalman filter and energy equilibrium theory, a new method can be proposed to solve the problem of detecting damage for such largesize structural systems mentioned above. The Kalman filter provides a recursive solution of discrete linear data (Kalman, 1960). In recent years, some observerbased estimation techniques have been extensively studied by many researchers, including the least squares estimation (Groeneboom et al., 2001; Feng et al., 2008), the modified Kalman filter (Hoshiya and Saito, 1984; Ghanem and Shinozuka, 1995; Shinozuka and Ghanem, 1995; Yang et al., 2006; Song and Dyke, 2014), and the particle filter (van der Merwe et al., 2004; Chatzi and Smyth, 2009). On the other hand, energy-based structure analysis has been developed and implemented by many researchers since it was proposed. The energy equilibrium theory has already proved to be an effective basis for methods in structural engineering. However, dynamical damage identification strategies based on energy are still new topics (Xu and $\mathrm{Wu}, 2007)$. The energy damage identification strategy based on strain can locate damage positions accurately, and it can also quantify damage intensity and resist environmental noise (Xu et al., 2011).

To meet this demand, an in-time model updating approach is proposed to identify stiffness, find the location of damage, and quantify its intensity in structures which contain a large number of DOFs. This approach establishes a relationship between element stiffness and structural responses. Combining the energy equilibrium theory and the Kalman filter together, the structural stiffness can be obtained by comparing the predicted energy and measured energy in each step. A series of simulations on a highway sign support truss by this approach indicates that the unknown parameters can converge rapidly to the true value. In other words, the approach can locate the damage and measure the damage intensity in-time. The term 'in-time' in the study means the approach can promptly evaluate the damage, usually in less than $1 \mathrm{~s}$ in this study.

\section{Description of a full-scale highway sign support truss}

The real-world structure considered in the simulation is one segment of a sign support truss, formerly placed at Interstate I-29 near Sioux City in Iowa, USA as shown in Fig. 1 (Yan et al., 2012). This highway sign support truss was out of service and is now available for testing at the Robert L. and Terry L. Bowen Laboratory for Large-scale Civil Engineering Research at Purdue University, USA. The structure is a horizontal, 3D four-chord welded spatial reticulated truss. The horizontal space truss spans longitudinally 
$10.38 \mathrm{~m}$ with its four main chords placed on a $1.83 \mathrm{~m} \times 1.98 \mathrm{~m}$ configuration. The entire highway truss consisted of four segments; however, only one of them is analyzed in this study. All bars of the truss are made of round, tubular aluminum (6061-T6) rods.

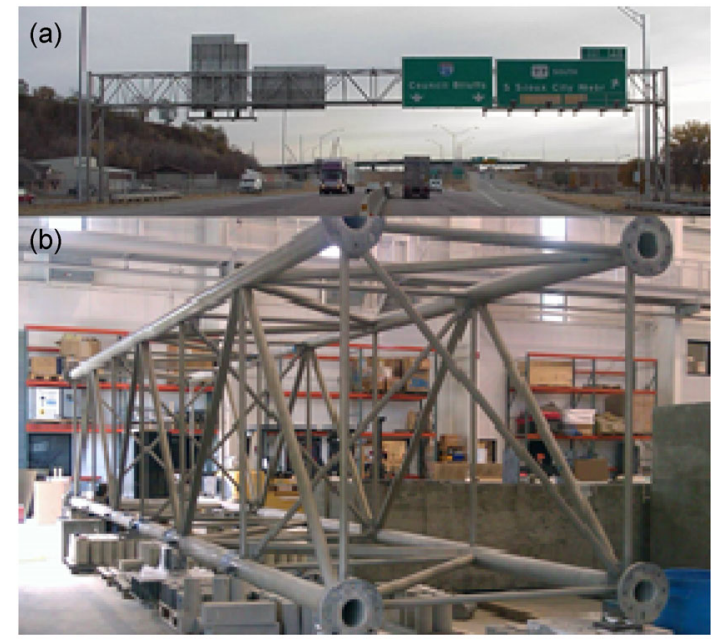

Fig. 1 Sign support structure over Interstate I-29 when it was in service (a) and one segment of the structure in Bowen Lab (b)

The structure was mounted with one end simply supported on metal blocks to simulate a pinned joint, and the other ends were placed on cylinders to simulate roller supports (Fig. 2) (Krishnan et al., 2011).
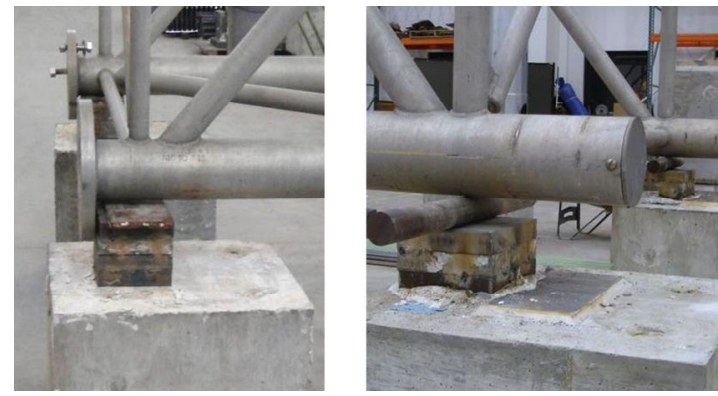

Fig. 2 Boundary condition of experimental setup

The truss was vibrated by a shaker in the $Z$ direction (vertical). Because environmental vibrations provide rich spectrum components on the sign support truss (such as wind, ground vibration, and vibration caused by traffic), the shaker was set to a band limited white noise of $0-200 \mathrm{~Hz}$. The noise level of the ambient was found to be of the order of $0.1 \mathrm{~g}$ $\left(g=9.8 \mathrm{~m} / \mathrm{s}^{2}\right)$, and the excitation of the shaker created an average $1 g$ level. The shaker was located adjacent to the middle node (Fig. 3). The white noise signal from the signal generator was first amplified through an amplifier before being sent to the electro-dynamic shaker. The baseline noise in the system was also recorded.
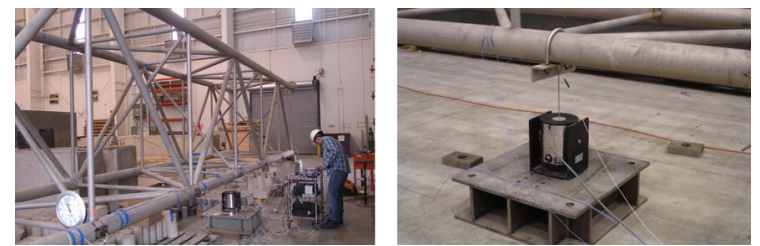

Fig. 3 Experimental setup of the Electro-dynamic shaker

The types of damage observed in the real-world are dents, tears, and cracks in regions away from the ends, weld cracks in diagonal member joints to the main chord, weld cracks at the joints of vertical members and main chord, and bolt loosening and splice gaps at the flange connections. To validate the damage identification approach, multiple damage instances were created. They were induced on the structure by a variety of cuts through the member so as to simulate the damage to the member, which are shown in Fig. 4.

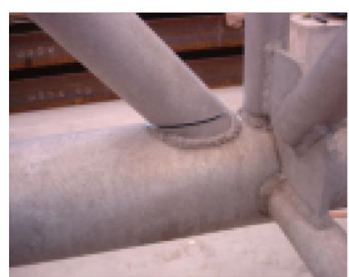

(a)

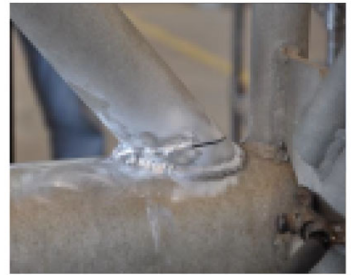

(c)

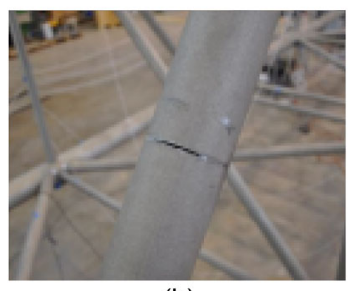

(b)

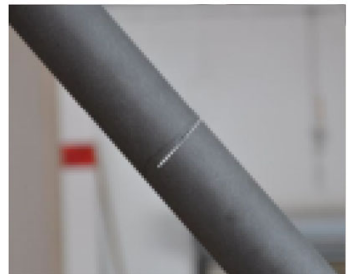

(d)
Fig. 4 Multiple damage cases

(a) Element 20, full cut; (b) Element 45, 20\% cut; (c) Element 64, 50\% cut; (d) Element 76, $80 \%$ cut 


\section{Theories of the damage identification method}

\subsection{Energy equilibrium equation of an elastic system}

For single degree of freedom (SDOF) system, if it is assumed that the damping depends on the velocity, a complete dynamic equilibrium equation of a SDOF system can be expressed as

$$
m \ddot{y}+c \dot{y}+k y=f,
$$

where $m$ is the mass, $c$ is the damping, $k$ is the stiffness, $y$ is the displacement of the particle, $\dot{y}$ is the velocity, $\ddot{y}$ is the acceleration, and $f$ is the input force of the SDOF system. Additionally, $y$ and $f$ are the functions with respect to time $t$. The displacement is assumed to be 0 when $t=0$, i.e., $\left.\dot{y}\right|_{t=0}=\left.\ddot{y}\right|_{t=0}=0$. If both sides of Eq. (1) are multiplied by velocity $\dot{y}$, and then integrated over time $t$, the energy equilibrium equation of the SDOF dynamic system at time $t$ can be expressed as

$$
\int_{0}^{t} m \dddot{y} \dot{y} \mathrm{~d} t+\int_{0}^{t} c \dot{y} \dot{y} \mathrm{~d} t+\int_{0}^{t} k y \dot{y} \mathrm{~d} t=\int_{0}^{t} f \dot{y} \mathrm{~d} t,
$$

where the first term is the kinetic energy of the SDOF system, i.e., $W_{\mathrm{ek}}=\int_{0}^{t} m \ddot{y} \dot{\mathrm{y}} \mathrm{d} t=\left.\frac{m}{2} \dot{y}^{2}\right|_{0} ^{t}$; the second term is the energy dissipated by damping, i.e., $W_{\mathrm{h}}=\int_{0}^{t} c \dot{y}^{2} \mathrm{~d} t$; the third term is strain energy, i.e., $W_{\text {es }}=\int_{0}^{t} k y \dot{y} \mathrm{~d} t=\left.\frac{k}{2} y^{2}\right|_{0} ^{t} ;$ and the right hand term of the equation is input energy, i.e., $W_{\mathrm{f}}=\int_{0}^{t} f \dot{y} \mathrm{~d} t$. The energy equilibrium equation of the SDOF system can be expressed as Eq. (3), which means that the internal energy is equal to the external energy:

$$
W_{\mathrm{ek}}+W_{\mathrm{h}}+W_{\mathrm{es}}=W_{\mathrm{f}} .
$$

For the multi degree of freedom (MDOF) system, the dynamic equilibrium equation of a MDOF system can be expressed as

$$
M \ddot{y}+C \dot{y}+K y=F,
$$

where $\boldsymbol{M}$ is the mass matrix of the dynamic system, $\boldsymbol{C}$ is the damping matrix of the system, $\boldsymbol{K}$ is the stiffness matrix, $\boldsymbol{y}$ is the vector of displacements, $\dot{\boldsymbol{y}}$ is the vector of velocities, $\ddot{\boldsymbol{y}}$ is the vector of accelerations, and $\boldsymbol{F}$ is the external force. The displacement and velocity of the system are assumed to be 0 when $t=0$, i.e., $\left.\dot{\boldsymbol{y}}\right|_{t=0}=\left.\ddot{\boldsymbol{y}}\right|_{t=0}=0$. If both sides of Eq. (4) are multiplied by $\dot{\boldsymbol{y}}^{\mathrm{T}}$ and then integrated over time $t$, the energy equation of the MDOF dynamic system at time $t$ can be expressed as

$$
\int_{0}^{t} \dot{\boldsymbol{y}}^{\mathrm{T}} \boldsymbol{M} \ddot{\boldsymbol{y}} \mathrm{d} t+\int_{0}^{t} \dot{\boldsymbol{y}}^{\mathrm{T}} \boldsymbol{C} \dot{\boldsymbol{y}} \mathrm{d} t+\int_{0}^{t} \dot{\boldsymbol{y}}^{\mathrm{T}} \boldsymbol{K} \boldsymbol{y} \mathrm{d} t=\int_{0}^{t} \dot{\boldsymbol{y}}^{\mathrm{T}} \boldsymbol{F}(t) \mathrm{d} t,
$$

where the first term of the left side represents the kinetic energy of the MDOF system at time $t$, i.e., $W_{\text {ek }}=\int_{0}^{t} \dot{\boldsymbol{y}}^{\mathrm{T}} \boldsymbol{M} \ddot{\boldsymbol{y}} \mathrm{d} t=\frac{1}{2} \dot{\boldsymbol{y}}^{\mathrm{T}} \boldsymbol{M} \dot{\boldsymbol{y}} ;$ the second term of the left side is the energy dissipated by the damping at time $t$, i.e., $W_{\mathrm{h}}=\int_{0}^{t} \dot{\boldsymbol{y}}^{\mathrm{T}} \boldsymbol{C} \boldsymbol{y} \mathrm{d} t$; the third term is the strain energy, i.e., $W_{\mathrm{es}}=\int_{0}^{t} \dot{\boldsymbol{y}}^{\mathrm{T}} \boldsymbol{K} \boldsymbol{y} \mathrm{d} t=\frac{1}{2} \boldsymbol{y}^{\mathrm{T}} \boldsymbol{K} \boldsymbol{y}$; and the right side of the equation is the input energy by the external force, i.e., $W_{\mathrm{f}}=\int_{0}^{t} \dot{\boldsymbol{y}}^{\mathrm{T}} \boldsymbol{F}(t) \mathrm{d} t$. Based on the energy equilibrium theory, the energy equilibrium of the MDOF system can be also expressed as

$$
\boldsymbol{W}_{\mathrm{ek}}+\boldsymbol{W}_{\mathrm{h}}+\boldsymbol{W}_{\mathrm{es}}=\boldsymbol{W}_{\mathrm{f}} .
$$

\subsection{Kalman filter algorithm}

The Kalman filter algorithm assumes the state $\boldsymbol{x} \in \mathbb{R}^{n}$ of a discrete-time process that is governed by the linear state space equation as

$$
\boldsymbol{x}_{j}=\boldsymbol{A} \boldsymbol{x}_{j-1}+B \boldsymbol{u}_{j-1}+\boldsymbol{w}_{j-1},
$$

with a measurement $\boldsymbol{z} \in \mathbb{R}^{n}$ that is

$$
\boldsymbol{z}_{j}=\boldsymbol{H} \boldsymbol{x}_{j-1}+\boldsymbol{v}_{j},
$$

where $\mathbb{R}^{n}$ is an $n$-dimensional state space, $\boldsymbol{A}$ is the state transition matrix, $\boldsymbol{B}$ is the input coupling matrix, 
and $\boldsymbol{H}$ is the measurement sensitivity matrix. The random variables $\boldsymbol{w}_{j}$ and $\boldsymbol{v}_{j}$ are the process noise and measurement noise, respectively. $\boldsymbol{u}_{j-1}$ is the optional control input, and the subscript $j$ is the $j$ th time step. The parameters $\boldsymbol{w}_{j}$ and $\boldsymbol{v}_{j}$ are assumed to be independent, and they have normal probability distributions as

$$
\begin{aligned}
& p(\boldsymbol{w}) \sim N(0, \boldsymbol{Q}), \\
& P(\boldsymbol{v}) \sim N(0, \boldsymbol{R}) .
\end{aligned}
$$

In real-world, the process noise covariance $Q$ and measurement noise covariance $\boldsymbol{R}$ might be timevarying. But we assume they are constant here. Then the a priori and a posteriori estimate errors can be defined as $\boldsymbol{e}_{j}^{-} \equiv \boldsymbol{x}_{j}-\hat{\boldsymbol{x}}_{j}^{-}$and $\boldsymbol{e}_{j} \equiv \boldsymbol{x}_{j}-\hat{\boldsymbol{x}}_{j}$, respectively. The a priori covariance matrix of state estimation uncertainty is defined as $\boldsymbol{P}_{j}^{-}=\boldsymbol{E}\left[\boldsymbol{e}_{j}^{-} \boldsymbol{e}_{j}^{-\mathrm{T}}\right]$, and the a posteriori covariance matrix of state estimation uncertainty is $\boldsymbol{P}_{j}=\boldsymbol{E}\left[\boldsymbol{e}_{j} \boldsymbol{e}_{j}^{\mathrm{T}}\right]$. Above all, the specific equations for the time and measurement updates are presented as equations below:

Discrete Kalman filter time update equations:

$$
\begin{aligned}
& \hat{\boldsymbol{x}}_{j}^{-}=\boldsymbol{A} \hat{\boldsymbol{x}}_{j-1}+\boldsymbol{B} \boldsymbol{u}_{j-1}, \\
& \boldsymbol{P}_{j}^{-}=\boldsymbol{A} \boldsymbol{P}_{j-1} \boldsymbol{A}^{\mathrm{T}}+\boldsymbol{Q} .
\end{aligned}
$$

Discrete Kalman filter measurement update equations:

$$
\begin{gathered}
\boldsymbol{K}_{j}=\boldsymbol{p}_{j}^{-} \boldsymbol{H}^{\mathrm{T}}\left(\boldsymbol{H} \boldsymbol{P}_{j}^{-} \boldsymbol{H}^{\mathrm{T}}+\boldsymbol{R}\right)^{-1}, \\
\hat{\boldsymbol{x}}_{j}=\hat{\boldsymbol{x}}_{j}^{-}+\boldsymbol{K}\left(\boldsymbol{z}_{j}-\boldsymbol{H} \hat{\boldsymbol{x}}_{j}^{-}\right), \\
\boldsymbol{P}_{j}=\left(\boldsymbol{I}-\boldsymbol{K}_{j} \boldsymbol{H}\right) \boldsymbol{P}_{j}^{-} .
\end{gathered}
$$

The Kalman gain $\boldsymbol{K}_{j}$ in Eq. (12) is the key point of the measurement update equation. After measuring the process $\boldsymbol{z}_{j}$, the next step is to actually calculate an a posteriori state estimate by incorporating the measurement as in Eq. (13). Then an a posteriori covariance matrix of state estimation uncertainty is obtained by Eq. (14). After importing an initial state $\hat{\boldsymbol{x}}_{0}$, a priori covariance matrix of state estimation uncertainty, $\boldsymbol{P}_{0}$ and the measurement noise covariance $\boldsymbol{R}_{0}$ are then calculated repeatedly via Eqs. (10)-(14) to obtain the eventual state $\boldsymbol{x}$.

\subsection{In-time damage identification approach}

To update the stiffness of the structure, a relationship between these unknown parameters and the structural energy is established. If the truss has $l$ elements (rods) and $n$ DOFs, the Young's modulus $\boldsymbol{E}=\left[E_{1}, E_{2}, \ldots, E_{i}, \ldots, E_{l}\right]^{\mathrm{T}}$ is chosen to represent the stiffness of the highway sign support truss, where $E_{i}$ is the Young's modulus of the $i$ th element in the structure. Firstly, we assume there is an 'initial structure', whose Young's modulus of each member in the structure is equal to $1.0 \times 10^{10} \mathrm{~N} / \mathrm{m}^{2}$, i.e., $\bar{E}=\left[\bar{E}_{1}\right.$, $\left.\bar{E}_{2}, \cdots, \bar{E}_{i}, \cdots, \bar{E}_{l}\right]^{\mathrm{T}}=[1,1, \ldots, 1, \ldots, 1]^{\mathrm{T}} \times 10^{10} \mathrm{~N} / \mathrm{m}^{2}$. The stiffness matrix of all the members of the "initial structure' can be defined as $\overline{\boldsymbol{K}}_{\mathrm{g}}=\left[\overline{\boldsymbol{K}}_{1}, \overline{\boldsymbol{K}}_{2}, \cdots, \overline{\boldsymbol{K}}_{i}, \cdots\right.$, $\left.\overline{\boldsymbol{K}}_{l}\right]^{\mathrm{T}}$, where $\overline{\boldsymbol{K}}_{i}$ is the stiffness matrix of the $i$ th element of the 'initial structure' in the global coordinate system. The stiffness matrix of the 'true structure' (unknown or real-world structure) of all the members can be defined as $\boldsymbol{K}_{\mathrm{g}}=\left[\boldsymbol{K}_{1}, \boldsymbol{K}_{2}, \ldots, \boldsymbol{K}_{i}, \ldots\right.$, $\left.\boldsymbol{K}_{l}\right]^{\mathrm{T}}$, and $\boldsymbol{K}_{i}$ is the stiffness matrix of element $i$ with a true Young's modulus in the global coordinate system. Thus, $\boldsymbol{K}_{i}=E_{i} \overline{\boldsymbol{K}}_{i}$ is the real stiffness matrix of the element $i$. The strain energy $\overline{\boldsymbol{W}}_{\text {es }}$ of the initial structure' subject to the response of the 'true structure' can be expressed as

$$
\begin{aligned}
& \overline{\boldsymbol{W}}_{\mathrm{es}}=\int_{0}^{t} \boldsymbol{v}_{\mathrm{g}}^{\mathrm{T}} \overline{\boldsymbol{K}}_{\mathrm{g}} \boldsymbol{u} \mathrm{d} t \\
& =\int_{0}^{t}\left[\begin{array}{ccccc}
\boldsymbol{v}^{\mathrm{T}} & & & & \\
& \ddots & & & \\
& & \boldsymbol{v}^{\mathrm{T}} & & \\
& & & \ddots & \\
& & & & \boldsymbol{v}^{\mathrm{T}}
\end{array}\right]\left[\begin{array}{c}
\overline{\boldsymbol{K}}_{1} \\
\vdots \\
\overline{\boldsymbol{K}}_{i} \\
\vdots \\
\overline{\boldsymbol{K}}_{l}
\end{array}\right]\left\{\begin{array}{c}
u_{1} \\
\vdots \\
u_{i} \\
\vdots \\
u_{n}
\end{array}\right\} \mathrm{d} t,
\end{aligned}
$$

where $\boldsymbol{v}_{\mathrm{g}}$ is the $(l \times n) \times n$ matrix; $\boldsymbol{v}$ is the $n \times 1$ velocity vector, i.e., $\boldsymbol{v}=\left[v_{1}, v_{2}, \ldots, v_{i}, \ldots, v_{n}\right]^{\mathrm{T}}$, and $v_{i}$ is the velocity of the $i$ th DOF; $\overline{\boldsymbol{K}}_{\mathrm{g}} \in \mathbb{R}^{(m \times n) \times n}$ is the stiffness of 
the 'initial structure', $\overline{\boldsymbol{K}}_{i} \in \mathbb{R}^{n \times n}$ is the stiffness matrix in global coordinate system; and $\boldsymbol{u} \in \mathbb{R}^{n \times 1}$ is the displacement vector of all the DOFs. The size of the $(l \times n) \times n$ stiffness matrix $\overline{\boldsymbol{K}}_{\mathrm{g}}$ is much larger than the normal $n \times n$ global stiffness $\overline{\boldsymbol{K}}_{i}$. The reason for this assembly of matrix $\overline{\boldsymbol{K}}_{\mathrm{g}}$ is that, it can easily produce an $l \times 1$ strain energy vector $\overline{\boldsymbol{W}}_{\text {es }}$ of each member. The strain energy $W_{\text {es }}$ and $\overline{\boldsymbol{W}}_{\text {es }}$ are the key parameters for the damage identification algorithm. These two measured parameters are compared in each time step to produce an updated system state, i.e., Young's modulus $\boldsymbol{E}$ of the truss. In this study, with the help of Eq. (15), an $l \times 1$ strain energy vector $\overline{\boldsymbol{W}}_{\text {es }}$ of each member is obtained from $\overline{\boldsymbol{K}}_{\mathrm{g}}=\left[\overline{\boldsymbol{K}}_{1}, \overline{\boldsymbol{K}}_{2}, \cdots, \overline{\boldsymbol{K}}_{i}, \cdots\right.$, $\left.\overline{\boldsymbol{K}}_{l}\right]^{\mathrm{T}}$. By using the constantly updated parameter $W_{\mathrm{es} j}$ in Eq. (21), the measurement update equations of Kalman filter can be operative and produce an updated Young's modulus $\boldsymbol{E}_{j}$ at each time step. The kinetic energy can be expressed as

$$
W_{\mathrm{ek}}=\int_{0}^{t} \boldsymbol{v}^{\mathrm{T}} \boldsymbol{M a} \mathrm{d} t
$$

where $\boldsymbol{M}$ is the $n \times n$ global mass matrix of the 'true structure', and $\boldsymbol{a}$ is the $n \times 1$ acceleration vector of the structure. The energy dissipated by damping can be expressed as

$$
W_{\mathrm{h}}=\int_{0}^{t} \boldsymbol{v}^{\mathrm{T}} \boldsymbol{C} \boldsymbol{v} \mathrm{d} t
$$

where $\boldsymbol{C}$ is the $n \times n$ damping matrix of the 'true structure', and $\boldsymbol{v}$ is the $n \times 1$ velocity vector of the structure. The input energy caused by the shaker can be expressed as $W_{\mathrm{f}}=\int_{0}^{t} \boldsymbol{v}^{\mathrm{T}} \boldsymbol{F} \mathrm{d} t$. The strain energy of the 'true structure' can be obtained by

$$
W_{\mathrm{es}}=W_{\mathrm{f}}-W_{\mathrm{ek}}-W_{\mathrm{h}} .
$$

The deference between $\bar{W}_{\text {es }}$ and $W_{\text {es }}$ is caused by the inequality of the 'initial structure' and the 'true structure'.
Above all, the discrete-time state equations for the model updating approach can be expressed as

$$
\begin{gathered}
\boldsymbol{E}_{j}=\boldsymbol{I}_{j-1}+\boldsymbol{w}_{j}, \\
W_{\mathrm{es} j}=\overline{\boldsymbol{W}}_{\mathrm{es}}^{\mathrm{T}} \boldsymbol{E}_{j-1}+v_{j},
\end{gathered}
$$

where $\boldsymbol{E}_{j-1}$ is the Young's modulus at time step $j-1$, and $\boldsymbol{E}_{j}$ is the Young's modulus at time step $j ; \boldsymbol{I}$ is the identity matrix and is referred to the matrix $\boldsymbol{A}$ in Eq. (10); $W_{\text {es } j}$ is the total strain energy at time step $j$, $\overline{\boldsymbol{W}}_{\mathrm{es}}$ is the strain energy of the 'initial structure' whose Young's modulus is $1 \times 10^{10} \mathrm{~N} / \mathrm{m}^{2}$, so $\overline{\boldsymbol{W}}_{\mathrm{es}}$ is constant; and $\boldsymbol{w}_{j}$ and $\boldsymbol{v}_{j}$ are the noise of the system process and measurement, respectively. So the core equations of the proposed model updating approach are as follows:

The time update equations are

$$
\begin{aligned}
\hat{\boldsymbol{E}}_{j}^{-} & =\boldsymbol{I} \hat{\boldsymbol{E}}_{j-1}, \\
\boldsymbol{P}_{j}^{-} & =\boldsymbol{I} \boldsymbol{P}_{j-1} \boldsymbol{I}^{\mathrm{T}},
\end{aligned}
$$

and the measurement update equations are

$$
\begin{aligned}
& \boldsymbol{K}_{j}=\boldsymbol{P}_{j}^{-} \overline{\boldsymbol{W}}_{\mathrm{es}}\left(\overline{\boldsymbol{W}}_{\mathrm{es}} \boldsymbol{P}_{j}^{-} \overline{\boldsymbol{W}}_{\mathrm{es}}^{\mathrm{T}}+R\right)^{-1}, \\
& \hat{\boldsymbol{E}}_{j}=\hat{\boldsymbol{E}}_{j}^{-}+\boldsymbol{K}_{j}\left(W_{\mathrm{es} j}-\overline{\boldsymbol{W}}_{\mathrm{es}} \hat{\boldsymbol{E}}_{j}^{-}\right), \\
& \boldsymbol{P}_{j}=\left(\boldsymbol{I}-\boldsymbol{K}_{j} \overline{\boldsymbol{W}}_{\mathrm{es}}\right) \boldsymbol{P}_{j}^{-},
\end{aligned}
$$

where $\hat{\boldsymbol{E}}_{j}^{-}$is the a priori Young's modulus estimate at time step $j$, and $\hat{\boldsymbol{E}}_{j}$ is the a posteriori Young's modulus estimate at time step $j ; \boldsymbol{P}_{j}^{-}$is the a priori error covariance estimate of Young's modulus at time step $j, \boldsymbol{P}_{j}$ is the a posteriori error covariance estimate at time step $j, \boldsymbol{K}_{j}$ is the Kalman gain at time step $j, R$ is the noise of measurement, $W_{\text {es } j}$ is the total strain energy of the 'true structure' at time step $j$ obtained by Eq. (18); $\overline{\boldsymbol{W}}_{\mathrm{es}}$ is the strain energy calculated by the response of the 'initial structure'. After obtaining the main equation of the proposed damage identification approach, the unknown Young's modulus can be updated through these equations. 


\section{Simulation verification of the damage identification approach}

\subsection{Simulation setup}

A finite element (FE) model of the real-world sign support truss was built and simulated in MATLAB, and it consists of 28 nodes and 83 elements. The nodes are numbered in Fig. 5. The model is rigid at connections between main chords and other members. Besides the four nodes constrained as boundary condition, each node has six DOFs, including three translational motions and three rotational motions. Each element is taken as a beam element in the FE model. The geometry and physical information of each member and the total structure are listed in Table 1. The length of bays 1-6 is equal to $1.73 \mathrm{~m}$. The truss is $1.83 \mathrm{~m}$ wide and $1.98 \mathrm{~m}$ high.

A $Z$-direction band-limited white noise $(0$ $200 \mathrm{~Hz}$ ) on node 4 is used to excite the truss in each test, and the responses in the three directions $(X, Y$, and $Z$ in Fig. 5) at all nodes are measured correspondingly. The generated white noise is imposed on the truss using an electro-dynamic shaker which is placed at node 4 (Fig. 5). The sampling frequency is $256 \mathrm{~Hz}$ when measuring the dynamical responses. All the above testing processes of model updating are simulated in MATLAB.

In each numerical test, acceleration responses in three directions at every node are recorded to make the following calculation. The procedure of the model updating approach involves three parts: First, the input energy $E_{A}$ of the true model (true model of the physical specimen) is calculated through input force. Then, the total energy $E_{B}$ of the initial model (model to be updated with false Young's modulus) is calculated by the previously obtained acceleration. Finally, $E_{A}$ and $E_{B}$ are substituted into the Kalman filter algorithm to produce the true Young's modulus. Note that both the true model and initial model are simulated in MATLAB.

\subsection{Simulation verification on an undamaged structure}

The mathematical procedures for detecting damage using the damage identification approach involve two parts. First, the strain energy of the structure is obtained via Eq. (18). Second, that energy is substituted into the Kalman filter via Eqs. (20) and (21). Then the updated structural Young's modulus can be obtained for each time step. Specifically, a time-history analysis is performed to get the structural response through SIMULINK using a $1.5 \mathrm{~s}$ long, $0-200 \mathrm{~Hz}$ band-limited wide noise as the input signal, where the time step is $1 / 5120 \mathrm{~s}$. Then the energy of the structure is calculated by responses from that

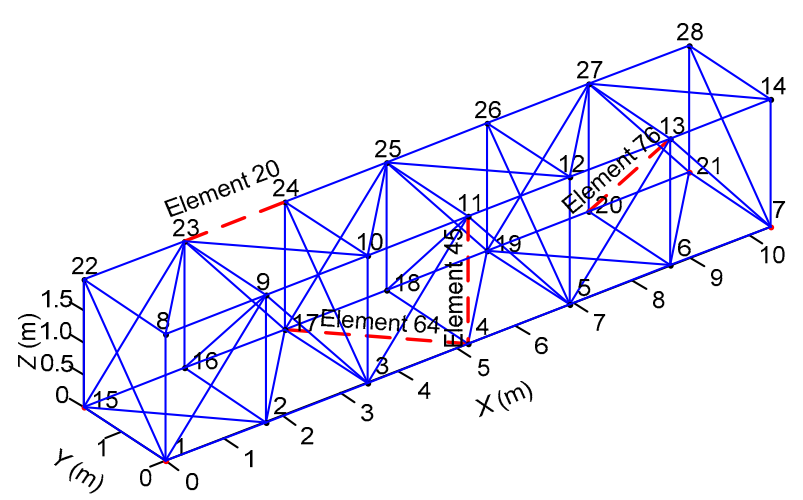

Fig. 5 Finite element model for the 3D truss in MATLAB and the location of damaged members

Table 1 Geometrical and material properties of the truss

\begin{tabular}{clr||lc}
\hline & Parameter & Value & Parameter & Value \\
\hline Main chords & Outside diameter $(\mathrm{mm})$ & 152.4 & Span of each bay in the left & 1.73 \\
& Thickness $(\mathrm{mm})$ & 79.0 & segment $(\mathrm{m})$ & \\
Diagonal braces & Outside diameter $(\mathrm{mm})$ & 76.2 & Span of each bay in the right & 1.64 \\
& thickness $(\mathrm{mm})$ & 6.3 & segment $(\mathrm{m})$ & 17.24 \\
End vertical braces & Outside diameter $(\mathrm{mm})$ & 63.5 & Total span length $(\mathrm{m})$ & $6.9625 \times 10^{10}$ \\
and end struts & Thickness $(\mathrm{mm})$ & 6.3 & Young's modulus $\left(\mathrm{N} / \mathrm{m}^{2}\right)$ & 2714.47 \\
Other members & Outside diameter $(\mathrm{mm})$ & 50.8 & Density $\left(\mathrm{kg} / \mathrm{m}^{3}\right)$ & 0.33 \\
& Thickness $(\mathrm{mm})$ & 4.8 & Poisson's ratio & \\
\hline
\end{tabular}


analysis, and the Kalman filter is used to estimate the Young's modulus of the structure, where the sampling frequency is $256 \mathrm{~Hz}$. It is indicated that the damage identification method needs a relativity low sampling frequency. The velocity and displacement of the truss are obtained via the integration and double integration of acceleration over time $t$.

It is assumed that all the parameters are known except the Young's modulus of the structure, i.e., the mass and damping ratio of the truss are assumed to be equal to those of the physical specimen. To begin with the calculation, the initial Young's modulus $\overline{\boldsymbol{E}}$ is assumed to be $1.0 \times 10^{10} \mathrm{~N} / \mathrm{m}^{2}$. The initial a priori covariance matrix $\boldsymbol{P}_{j}^{-}$is set as $1.0 \times 10^{8} \boldsymbol{I}$, where $\boldsymbol{I}$ is the $l \times l$ identity matrix. The initial measurement noise covariance $R$ is assigned as $1.0 \times 10^{-45}$. The strain energy of the 'initial structure' and 'true structure', $\bar{W}$ and $W_{\mathrm{es} j}$, can be calculated through Eq. (15) and Eq. (18). Then $\overline{\boldsymbol{E}}, R, \overline{\boldsymbol{W}}$, and $W_{\mathrm{es} j}$ are substituted into Eqs. (20) and (21) to start the calculation process of the Kalman filter. The results indicate that all the parameters of all the elements will converge to the true value, which is $6.9625 \times 10^{10} \mathrm{~N} / \mathrm{m}^{2}$. As shown in Fig. 6 , element 1 converges to the true value in $0.24 \mathrm{~s}$ (about 60 time steps); element 10, element 50, and element 83 converge to the true value in about $0.32 \mathrm{~s}$ (about 80 time steps); and the convergence conditions
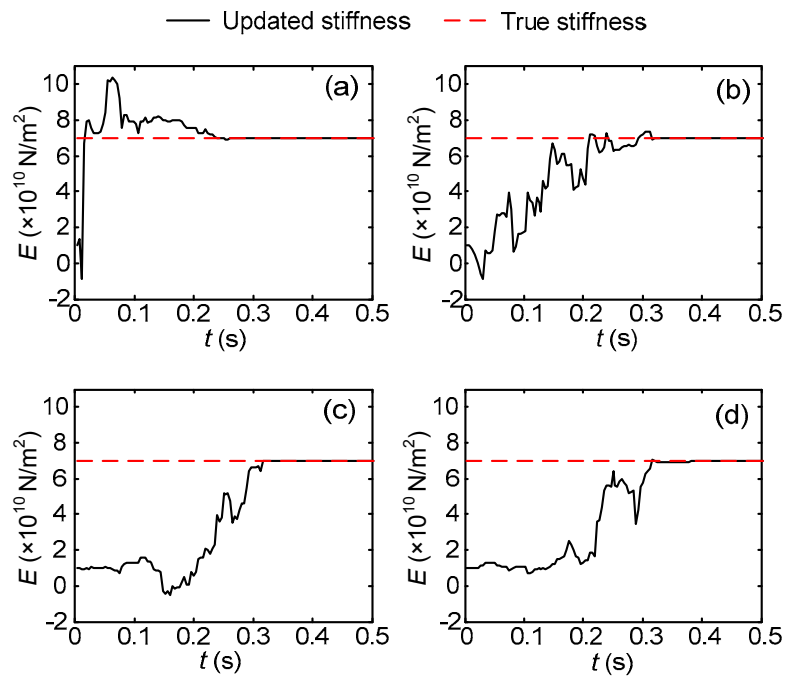

Fig. 6 Process of the damage identification approach for an undamaged structure

(a) Element 1; (b) Element 10; (c) Element 50; (d) Element 83 of the other elements are similar. The implication is that this damage identification approach can identify the Young's modulus and indicate the health of the structure in a short time.

\subsection{Simulation verification on damaged structures}

\section{Damage Case 1}

In this case, only one member (bar) at top of the back-panel in bay 2 (element 20, connecting joint 23 and joint 24 in Fig. 5) is assumed to lose all the stiffness to simulate the full cut through the section. By using the same method as for the undamaged truss, the results are shown in Fig. 7. It is shown in Fig. 7 that the Young's modulus of both damaged and healthy elements can converge to the true value. The Young's modulus of the initial truss for model updating is $1.0 \times 10^{10} \mathrm{~N} / \mathrm{m}^{2}$. As shown in Fig. 7, the damaged element 20 converges to the true value, $6.9625 \times 10^{10} \mathrm{~N} / \mathrm{m}^{2}$, in $0.15 \mathrm{~s}$ (about 51 time steps). The healthy element 1 , element 10 , and element 83 converges to the true value in about $0.28 \mathrm{~s}$ (about 72 time steps), $0.32 \mathrm{~s}$ ((about 82 time steps), and $0.34 \mathrm{~s}$ (about 87 time steps), respectively; all the other healthy elements of the truss can also converge to the true value, $6.9625 \times 10^{10} \mathrm{~N} / \mathrm{m}^{2}$, in $0.4 \mathrm{~s}$.
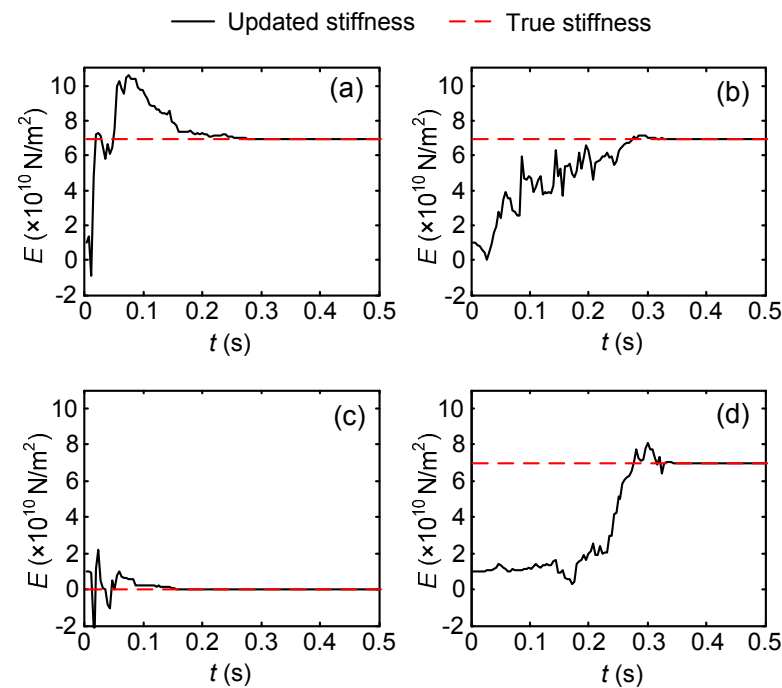

Fig. 7 Process of the damage identification approach of damage Case 1

(a) Element 1; (b) Element 10; (c) Element 20; (d) Element 83

\section{Damage Case 2}

In this case, three different elements (bars) are subjected to different levels of damages in the 
simulation. A bottom diagonal bar at bay 3 (element 45 , connecting joint 4 and joint 17) loses $20 \%$ element stiffness to simulate a cut of a fifth in depth of the full cross-section. A bar at front of panel 3 (element 64, connecting joint 4 and joint 11 ) loses $50 \%$ stiffness to simulate a halfway cut, and the middle diagonal at panel 6 (element 76, connecting joint 13 and joint 20) loses $80 \%$ stiffness to simulate an $80 \%$ cut. By using the same model updating approach as before, the Young's modulus of all the elements are calculated, and the results of four representative members are plotted in Fig. 8. For example, the healthy member, element 1 , converges from the initial value $1.0 \times 10^{10} \mathrm{~N} / \mathrm{m}^{2}$ to the true value $6.9625 \times 10^{10} \mathrm{~N} / \mathrm{m}^{2}$ in $0.25 \mathrm{~s}$ (about 64 time steps); the damaged member, element 45 , converges to its true value of $5.57 \times$ $10^{10} \mathrm{~N} / \mathrm{m}^{2}$ in $0.32 \mathrm{~s}$ (about 82 time steps), which means a $20 \%$ stiffness loss of the element; the damaged element 64 converges to its true value of $3.48 \times 10^{10} \mathrm{~N} / \mathrm{m}^{2}$ in $0.32 \mathrm{~s}$ (about 82 time steps), which means a $50 \%$ stiffness loss of the element; and the damaged element 76 converges to its true value of $1.39 \times 10^{10} \mathrm{~N} / \mathrm{m}^{2}$ in about $0.32 \mathrm{~s}$, which means an $80 \%$ stiffness loss of this element. The other healthy elements converge to the true value of $6.9625 \times$ $10^{10} \mathrm{~N} / \mathrm{m}^{2}$ in $0.4 \mathrm{~s}$. It is clear that the damage identification approach can identify the Young's modulus of the damaged part of the structure in a short time.
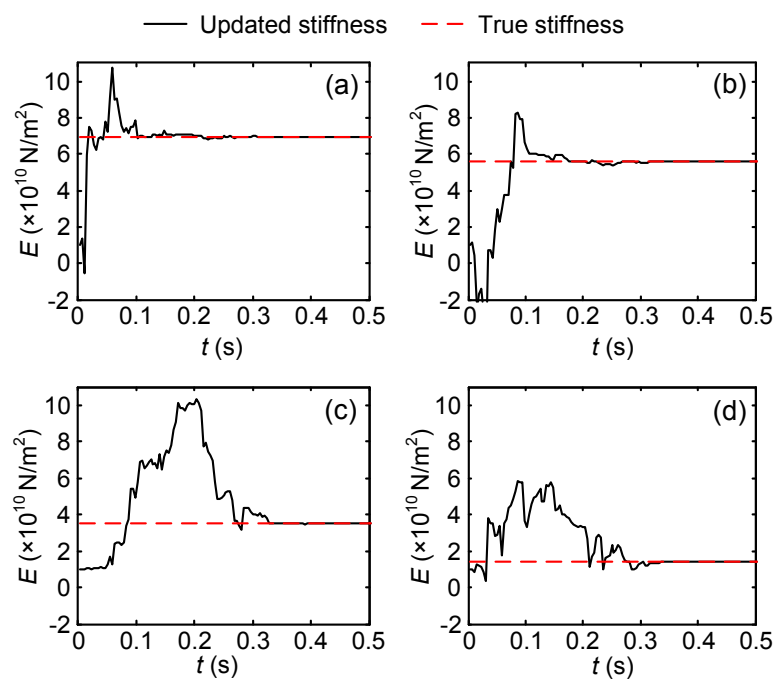

Fig. 8 Process of the damage identification approach of damaged Case 2

(a) Element 1; (b) Element 45; (c) Element 64; (d) Element 76

\subsection{Simulation verification on damaged structures with noise}

Noise with root mean square (RMS) noise-tosignal ratio at $5 \%$ is considered at all channels of the accelerometers. The identification process of the Young's modulus of element 20 and the three damaged elements is shown in Fig. 9. As shown in Fig. 9, although the convergence speed and precision are different for different elements, parameters of the damaged elements converge to the true value with only slight errors. The initial value of Young's modulus is $1 \times 10^{10} \mathrm{~N} / \mathrm{m}^{2}$, and the true value is $6.9625 \times$ $10^{10} \mathrm{~N} / \mathrm{m}^{2}$. At the end of $5 \mathrm{~s}$, the results of these four elements are as below: the Young's modulus of element 1 is $6.8675 \times 10^{10} \mathrm{~N} / \mathrm{m}^{2}$; the Young's modulus of element 45 is $5.4551 \times 10^{10} \mathrm{~N} / \mathrm{m}^{2}$; the Young's modulus of element 64 is $2.9622 \times 10^{10} \mathrm{~N} / \mathrm{m}^{2}$; and the Young's modulus of element 76 is $1.6136 \times 10^{10} \mathrm{~N} / \mathrm{m}^{2}$. The corresponding errors of these four elements are $1.30 \%, 2.06 \%, 14.91 \%$, and $15.87 \%$. So this model updating approach is stable in a noisy environment, specifically the average error is $8.55 \%$ and the maximum error is $15.87 \%$.
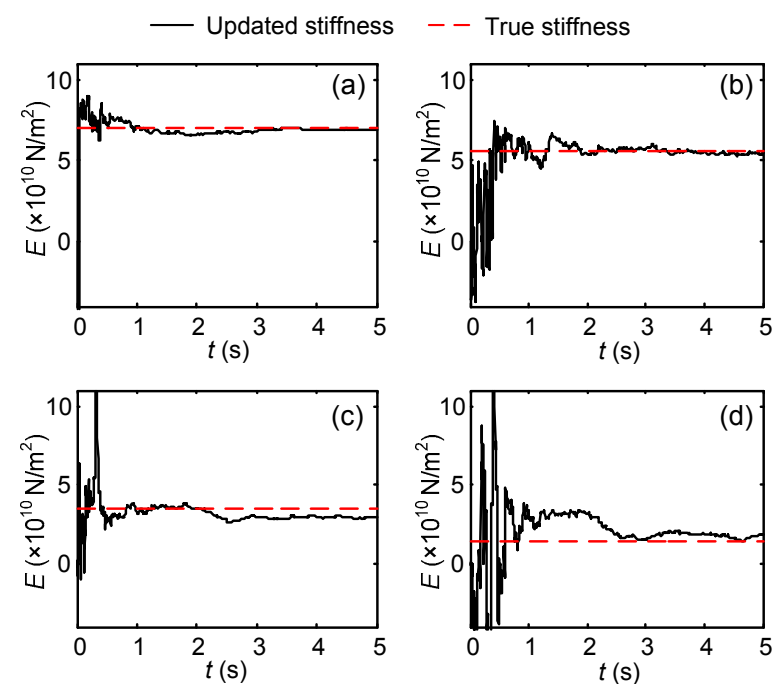

Fig. 9 Process of the damage identification approach with 5\% RMS noise

(a) Element 1; (b) Element 45; (c) Element 64; (d) Element 76

\subsection{Evaluation of the time required to implement the proposed "in-time" approach}

Compared to traditional health monitoring methods, the proposed damage identification 
approach, based on the Kalman filter and energy equilibrium theory, has yet another advantage, which is the ability to detect the damage in-time. This damage identification approach gathers structural acceleration responses and input force at every measured time step and produces the desired results immediately. The approach implemented substitutes these data in the energy equilibrium theory and the Kalman filter to get conclusions concerning such steps. As a result, the user is able to obtain structural stiffness information during the loading period and can also observe the trend behavior in the stiffness changes. This combination of observations is very important for predicting and preventing disasters.

The required time for the calculation process of this method involves two components. The first component is for the energy calculation; the second component is for the Kalman filter. Both computations are performed in MATLAB, in which different embedded programs can be used to get the required time for the damage identification approach in each step. The results are shown in Fig. 10, in which the horizontal straight lines are the sampling period, i.e., $1 / 256 \mathrm{~s}$. The computer used for the calculation was an Intel $^{\circledR}$ Core $^{\mathrm{TM}}$ i5-2310 processor, whose clock speed is $2.9 \mathrm{GHz}$ and Max turbo Frequency is $3.2 \mathrm{GHz}$, and a 4 GB DDR3-1066 Memory. Even under the same conditions, there are always slight differences (less than $1 \%$ ) of the required time of a MATLAB program running at different times. Even though such tiny differences were found, the conclusions are not affected. So only one representative trial is illustrated here. For example, in the undamaged case, the total time cost is $0.4628 \mathrm{~s}$, and the average time cost in
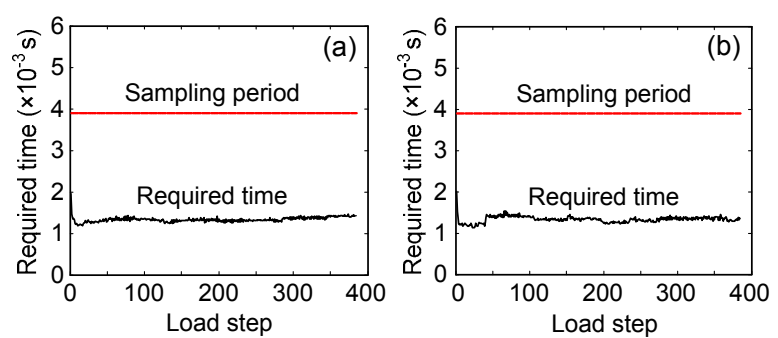

Fig. 10 Comparison between the time required of the undamaged truss (a) and the damaged truss (b) for each time step and sampling period every time step is about $0.0012 \mathrm{~s}$. In Case 2, the total required time is $0.4811 \mathrm{~s}$, and the average required time in each measured step is $0.0012 \mathrm{~s}$. As shown in Fig. 10, the time spent in each step is always shorter than the sampling period, which means that the damage identification approach is fast enough to update the model and detect the damage in real time.

\section{Conclusions}

A damage identification approach is proposed to identify the location of damage and to quantify its intensity. Numerical tests are conducted on a sign support truss structure to validate the proposed method. The conclusions of the proposed method are drawn as follows:

1. Numerical results demonstrate that the proposed damage identification approach can accurately locate the damage at a bar-level and measure the damage intensity. Additionally, the approach has a good performance on both single and multiple damage scenarios.

2. With the help of the damage identification approach, it was possible to get the previously unknown parameters to converge to the true value on time. The main advantage found during the implementation of this approach is that it is significantly time saving compared to others. This approach uses the Kalman filter, which provides an efficient computational means to estimate the state of a process. Taking the sign support truss for example, the element's Young's modulus of both a healthy and a damaged truss can converge to the true value in less than $0.4 \mathrm{~s}$, which is equal to 102 measured steps.

3. The damage identification approach is a method intended to offer a real time result. The time required in each measured step is considerably shorter than the sampling period, which makes the approach in-time or on-line. Taking the sign support truss as an example, the average time required for each step is $0.0012 \mathrm{~s}$, which is less than a third of the sampling period $1 / 256(0.0039) \mathrm{s}$.

4. The damage identification approach is also effective in a noisy environment. In this study, no error in calculating the Young's modulus of damaged elements is greater than $16 \%$ of the true value. 


\section{References}

Chatzi, E.N., Smyth, A.W., 2009. The unscented Kalman filter and particle filter methods for nonlinear structural system identification with non-collocated heterogeneous sensing. Structural Control \& Health Monitoring, 16(1):99-123. [doi:10.1002/stc.290]

Erdogan, Y.S., Bakir, P.G., 2013. Evaluation of the different genetic algorithm parameters and operators for the finite element model updating problem. Computers and Concrete, 11(6):541-569. [doi:10.12989/cac.2013.11.6. 541]

Feng, X.L., He, G.L., Abdurishit, 2008. Estimation of parameters of the Makeham distribution using the least squares method. Mathematics and Computers in Simulation, 77(1): 34-44. [doi:10.1016/j.matcom.2007.01.009]

Garcia-Perez, A., Amezquita-Sanchez, J.P., DominguezGonzalez, A., et al., 2013. Fused empirical mode decomposition and wavelets for locating combined damage in a truss-type structure through vibration analysis. Journal of Zhejiang University-SCIENCE A (Applied Physics \& Engineering), 14(9):615-630. [doi:10.1631/jzus.A1300030]

Ghanem, R., Shinozuka, M., 1995. Structural-system identification. I: Theory. Journal of Engineering Mechanics, 121(2):255-264. [doi:10.1061/(ASCE)0733-9399(1995) $121: 2(255)]$

Groeneboom, P., Jongbloed, G., Wellner, J.A., 2001. Estimation of a convex function: characterizations and asymptotic theory. Annals of Statistics, 29(6):1653-1698. [doi:10. 1214/aos/1015345958]

Hoshiya, M., Saito, E., 1984. Structural identification by extended Kalman filter. Journal of Engineering Mechanics, 110(12):1757-1770. [doi:10.1061/(ASCE)0733-9399(1984) 110:12(1757)]

Iwasaki, A., Todoroki, A., Shimamura, Y., et al., 2004. Unsupervised structural damage diagnosis based on change of response surface using statistical tool. JSME International Journal Series A, 47(1):1-7. [doi:10.1299/jsmea.47.1]

Kalman, R.E., 1960. A new approach to linear filtering and prediction problems. Journal of Fluids Engineering, 82(1):35-45.

Khoo, L.M., Mantena, P.R., Jadhav, P., 2004. Structural damage assessment using vibration modal analysis. Structural Health Monitoring, 3(2):177-194. [doi:10.1177/1475921 704042680]

Kim, J.T., Ryu, Y.S., Cho, H.M., et al., 2003. Damage identification in beam-type structures: frequency-based method vs mode-shape-based method. Engineering Structures, 25(1):57-67. [doi:10.1016/S0141-0296(02)00118-9]

Krishnan, S.S., Sun, Z.X., Irfanoglu, A., et al., 2011. Evaluating the performance of distributed approaches for modal identification. Proceeding of SPIE 7981, Sensors and Smart Structures Technologies for Civil, Mechanical, and Aerospace Systems, San Diego, USA, p.79814M79814M. [doi:10.1117/12.882143]

Lee, Y.S., Chung, M.J., 2000. A study on crack detection using eigenfrequency test data. Computers \& Structures, 77(3): 327-342. [doi:10.1016/S0045-7949(99)00194-7]

Lei, Y., Wang, H.F., Shen, W.A., 2012. Update the finite element model of canton tower based on direct matrix updating with incomplete modal data. Smart Structures and Systems, 10(4-5):471-483. [doi:10.12989/sss.2012.10. 4_5.471]

Li, T.Y., Zhang, T., Liu, J.X., et al., 2004. Vibrational wave analysis of infinite damaged beams using structure-borne power flow. Applied Acoustics, 65(1):91-100. [doi:10. 1016/j.apacoust.2003.07.004]

Liu, Y., Duan, Z.D., 2012. Fuzzy finite element model updating of bridges by considering the uncertainty of the measured modal parameters. Science China Technological Sciences, 55(11):3109-3117. [doi:10.1007/s11431-0125009-0]

Liu, Y., Sun, H., Wang, D.J., 2013. Updating the finite element model of large-scaled structures using component mode synthesis technique. Intelligent Automation and Soft Computing, 19(1):11-21. [doi:10.1080/10798587.2013.77 1457]

López-Díez, J., Torrealba, M., Güemes, A., et al., 2005. Application of statistical energy analysis for damage detection in spacecraft structures. Key Engineering Materials, 293-294:525-532. [doi:10.4028/www.scientific.net/KEM. 293-294.525]

Nadauld, J.D., Pantelides, C.P., 2007. Rehabilitation of cracked aluminum connections with GFRP composites for fatigue stresses. Journal of Composites for Construction, 11(3):328-335. [doi:10.1061/(ASCE)1090-0268(2007)11: 3(328)]

Park, J.S., Stallings, J.M., 2006. Fatigue evaluations of variable message sign structures based on AASHTO specifications. KSCE Journal of Civil Engineering, 10(3):207-217. [doi:10.1007/BF02824063]

Park, N.G., Park, Y.S., 2005. Identification of damage on a substructure with measured frequency response functions. Journal of Mechanical Science and Technology, 19(10): 1891-1901. [doi:10.1007/BF02984268]

Sadr, M.H., Astaraki, S., Salehi, S., 2007. Improving the neural network method for finite element model updating using homogenous distribution of design points. Archive of Applied Mechanics, 77(11):795-807. [doi:10.1007/s00419007-0129-x]

Shinozuka, M., Ghanem, R., 1995. Structural systemidentification. II: experimental-verification. Journal of Engineering Mechanics, 121(2):265-273. [doi:10.1061/ (ASCE)0733-9399(1995)121:2(265)]

Sinha, J.K., Friswell, M.I., 2003. The use of model updating for reliable finite element modelling and fault diagnosis of structural components used in nuclear plants. Nuclear Engineering and Design, 223(1):11-23. [doi:10.1016/ S0029-5493(03)00037-2]

Song, W., Dyke, S., 2014. Real-time dynamic model updating of a hysteretic structural system. Journal of Structural 
Engineering, 140(3):04013082. [doi:10.1061/(ASCE)ST. 1943-541X.0000857]

van der Merwe, R., Wan, E., Julier, S.J., 2004. Sigma-point Kalman filters for nonlinear estimation and sensor fusion: applications to integrated navigation. Proceedings of the AIAA Guidance Navigation \& Control Conference, Providence, Rhode Island, USA, p.1735-1764. [doi:10. 2514/6.2004-5120]

Xu, Z.D., Wu, Z.S., 2007. Energy damage detection strategy based on acceleration responses for long-span bridge structures. Engineering Structures, 29(4):609-617. [doi:10. 1016/j.engstruct.2006.06.004]

Xu, Z.D., Liu, M., Wu, Z.S., et al., 2011. Energy damage detection strategy based on strain responses for long-span bridge structures. Journal of Bridge Engineering, 16(5): 644-652. [doi:10.1061/(ASCE)BE.1943-5592.0000195]

Yan, A.M., Golinval, J.C., 2005. Structural damage localization by combining flexibility and stiffness methods. Engineering Structures, 27(12):1752-1761. [doi:10.1016/j. engstruct.2005.04.017]

Yan, G.R., Dyke, S.J., Irfanoglu, A., 2012. Experimental validation of a damage detection approach on a full-scale highway sign support truss. Mechanical Systems and Signal Processing, 28:195-211. [doi:10.1016/j.ymssp. 2011.10.008]

Yang, J.N., Lin, S.L., Huang, H.W., et al., 2006. An adaptive extended Kalman filter for structural damage identification. Structural Control \& Health Monitoring, 13(4): 849-867. [doi:10.1002/stc.84]
Zhao, X., Sun, H.H., Zheng, Y.M., 2009. Identification and updating for the three-dimensional finite element model of a long span steel skybridge. Structural Design of Tall and Special Buildings, 18(6):625-646. [doi:10.1002/tal.504]

\section{中文概要}

题 目: 一种基于 Kalman 滤波和能量原理的实时损伤识 别方法

目 的: 建立一种损伤识别方法, 能够实时地监测多自由 度复杂结构中构件的损伤情况。

方 法: 1. 用能量原理对结构刚度进行解构, 建立结构单 元刚度和节点响应之间的关系; 2. 用 Kalman 滤 波原理分析结构刚度的预测值和测量值, 迅速对 结构的刚度进行识别 (图 6-9); 3. 对每一步计算 进行耗时监测, 确保算法的实时性 (图 10)。

结 论: 1. 该方法能够较准确地得到结构的刚度信息, 同 时得出损伤单元的损伤位置和损伤量; 并且收玫 速度快, 计算量小, 具有很强的实时性和抗噪能 力; 2. 对于本文的桁架结构, 所有杆件刚度均能 在 $0.4 \mathrm{~s}$ 内收敛, 平均每一荷载步计算时间约为 $0.0012 \mathrm{~s}$, 小于采样周期 $1 / 256 \mathrm{~s}$, 说明该方法可 以迅速、准确地对结构进行实时的监测。

关键词: 实时模型修正; 能量平衡原理; Kalman 滤波原理; 损伤识别; 健康监测 